\title{
Time Response Parameters of Fiber Bragg Gratings
}

\author{
A.A.P. Pohl*,1 and J. Canning ${ }^{2}$ \\ ${ }^{I}$ Graduate School on Electrical Engineering and Applied Informatics (CPGEI), Federal University of Technology- \\ Parana (UTFPR), Brazil \\ ${ }^{2}$ Optical Fibre Technology Centre, School of Chemistry, The University of Sydney, Australia
}

\begin{abstract}
We report on experimental results related to the time response of Fiber Bragg Gratings. From the experimental data it is possible to retrieve important parameters such as the response delay and the grating switching time, which are properties of relevance for understanding the operation of fast tuning devices.
\end{abstract}

\section{INTRODUCTION}

Fiber Bragg Gratings (FBG) have been in use for almost thirty years and since then they have been applied to many areas either as devices in telecommunications or as the fundamental element in sensor applications (Canning FLO 2007) [1]. The shift of the Bragg wavelength has been its most significant characteristic since it is related with the sensing of external stimulus such as strain and temperature. In many applications the analysis of the wavelength shift requires no consideration of time. However, some applications do require an understanding of the speed in which the wavelength shift occurs as this feature is important for the operation of fast devices. Given a system where temperature remains constant, the shift in wavelength is most often achieved by changing the strain applied to the grating. Moreover, if the strain is time dependent, the shift in wavelength is described according to the expression

$\frac{\Delta \lambda_{B}(t)}{\lambda_{B}}=\gamma \varepsilon(t)$

where $\Delta \lambda_{B}$ is the Bragg wavelength shift, $\lambda_{B}$ is the Bragg wavelength of the FBG at rest, $\gamma$ is a parameter that is related to the fiber effective index, the components of the strain optic tensor and Poisson's ratio and $\varepsilon$ is the infinitesimal strain (Othonos and Kalli FBG 1999) [2].

The general theory of elasticity provides a physical explanation of motions that affect elastic bodies and is used to understand the propagation of strain waves in different materials. Particularly, due to its small diameter, an optical fiber may be considered as an elastic bar and the strain perceived by the fiber is best described by the elementary theory of longitudinal motion when the material transversal deformation is neglected (Eringen and Suhubi ELT 1975) [3]. This way, the description of the dynamic strain in a fiber being stretched by a longitudinal load, in which a grating has been written, can be adequately described by the expression

$c^{2} \frac{\partial^{2} u(x, t)}{\partial x^{2}}+F(x, t)=\frac{\partial^{2} u(x, t)}{\partial t^{2}}$

*Address correspondence to this author at the Federal University of Technology - Parana, UTFPR, Curitiba, PR, Brazil; Tel: ++55 413310 4695; Fax: ++ 55413310 4680; E-mail: pohl@utfpr.edu.br where $u$ is the displacement function, which is related to the one-dimensional strain by $\varepsilon_{x}=\partial u / \partial x$, c is the speed of the longitudinal wave strain propagating in the elastic bar and $F(x, t)$ is a given force function per mass unit that describes the behavior of friction forces in the material. This wave will cause a dynamic displacement in the bar changing its body volume.

In a previous work estimations were made concerning the speed in which the Bragg wavelength is shifted when the fiber is subjected to a longitudinal traction load (Neves Jr. JLT) [4]. In this work we present experimental results on the time response of gratings that are dynamically stretched along the longitudinal fiber axis by means of a piezoelectriccontrolled positioning stage. From the experimental data it is possible to retrieve important parameters such as the response delay and the grating switching time.

\section{EXPERIMENTAL ARRANGEMENT}

The basic idea of accessing the time response of a grating is to observe the time shift of the Bragg wavelength when the grating is stimulated by strain in the form of a step function. This can be accomplished by rapidly stretching the fiber in which the grating is written and observing the change in power with the help of a photo-detector and oscilloscope as the grating spectrum is moved from its rest position. The experimental set-up (Fig. 1) consists of two positioning stages, one of which is controlled by a piezoelectric element. A piece of fiber with the jacket stripped off is glued between the stages. Special care should be taken to position the grating close but outside the fixed stage. The other end of the fiber is glued on the stage which is controlled by the piezoelectric element. The quantity and type of glue is critical in avoiding effects from the glue itself. Square pulses of very low frequency and with a rise time of $40 \mu$ s and duration time of $10 \mathrm{~ms}$ are applied to the piezoelectric-driven stage by means of a pulse generator. The strain waveform produced by this method propagates in the fiber and reaches the grating on the other end causing the Bragg wavelength to shift. To access the time response, the line of a DFB tunable laser is positioned on the slope of the grating spectrum response (Tsuda CST 2006) [5].

As the grating spectrum is shifted by the strain pulse the DFB laser line moves causing the power in the photo- 


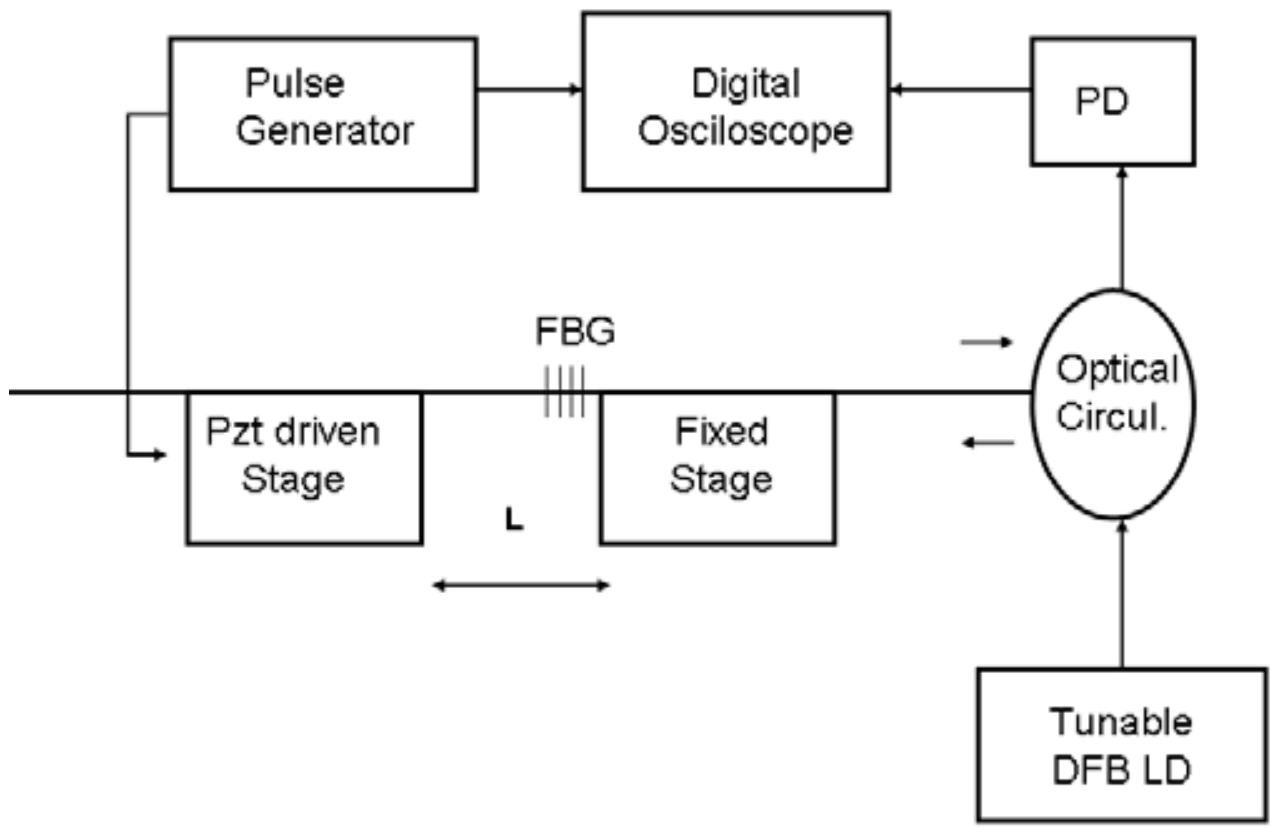

Fig. (1). Diagram of the experimental set-up. $\mathrm{L}$ is the length that represents the distance between the points where the fiber is glued.

detector to change. The signal is easily observed on the screen of an oscilloscope. Fig. (2) shows the temporal response of several measurements performed on the same piece of fiber, but adjusting to different lengths, L, between the two positioning stages.

The rectangular shape represents the electrical pulse waveform applied to the piezoelectric-driven stage and its rise time is taken as the reference for estimating the propagation time of the strain pulse till it reaches the grating. From the onset of the excitation waveform at $\mathrm{t}=0 \mathrm{~s}$ one observes the different times, associated with each length $\mathrm{L}$, that the strain pulse takes to reach the other end of the fiber where the grating is located. From these curves several features can be retrieved. First, it is remarkable to observe the different width of the optical response, which is associated with the strength applied to the grating at each L. The applied strain is estimated as $\Delta \mathrm{L} / \mathrm{L}$ and when the distance $\mathrm{L}$ increases, the strain seen by the grating is reduced. This causes the DFB line to be less displaced along the linear slope of the grating spectrum. This feature is best seen in the curves plotted in

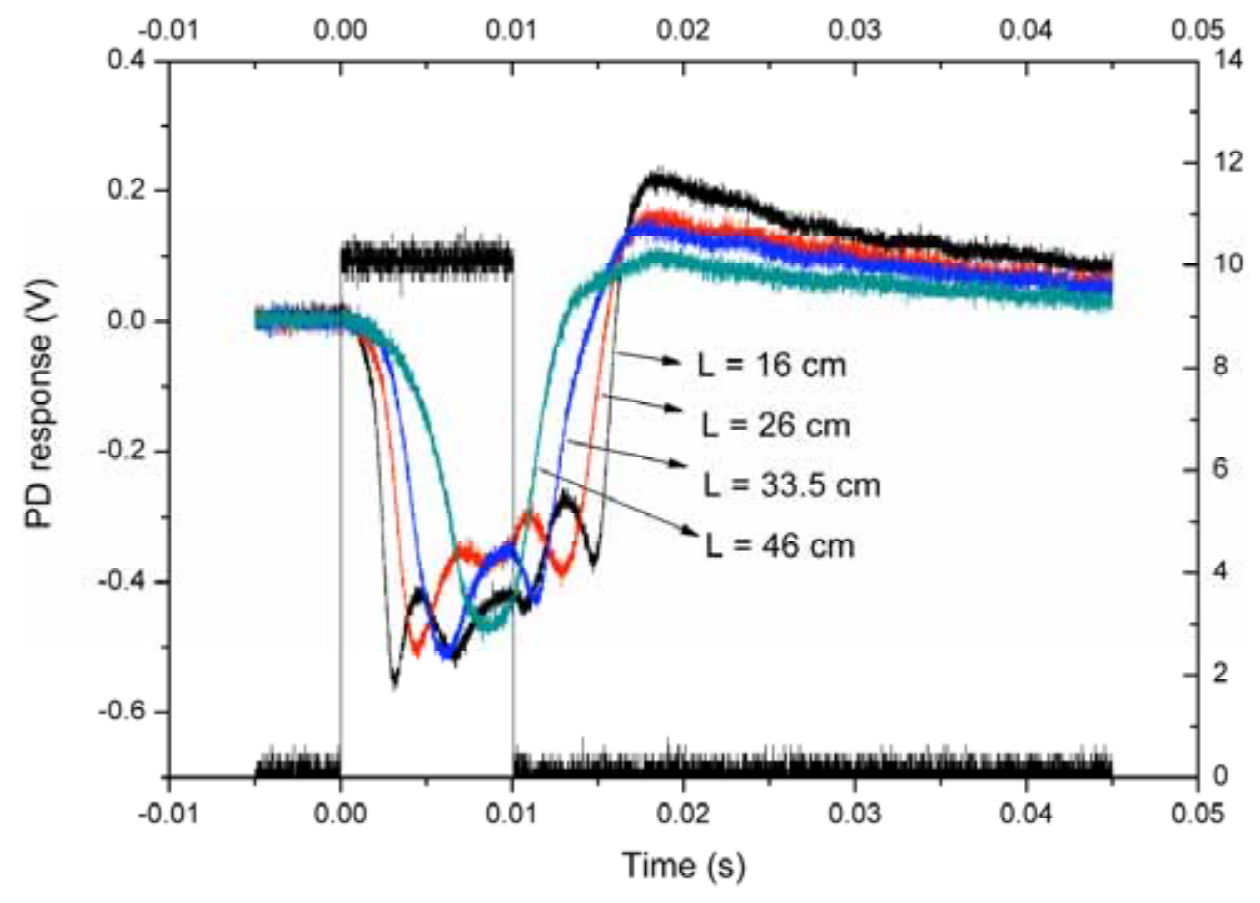

Fig. (2). Time response of a grating due to a square pulse applied to the piezoelectric driver. $\mathrm{L}$ is the length between the points where the grating is fixed. 


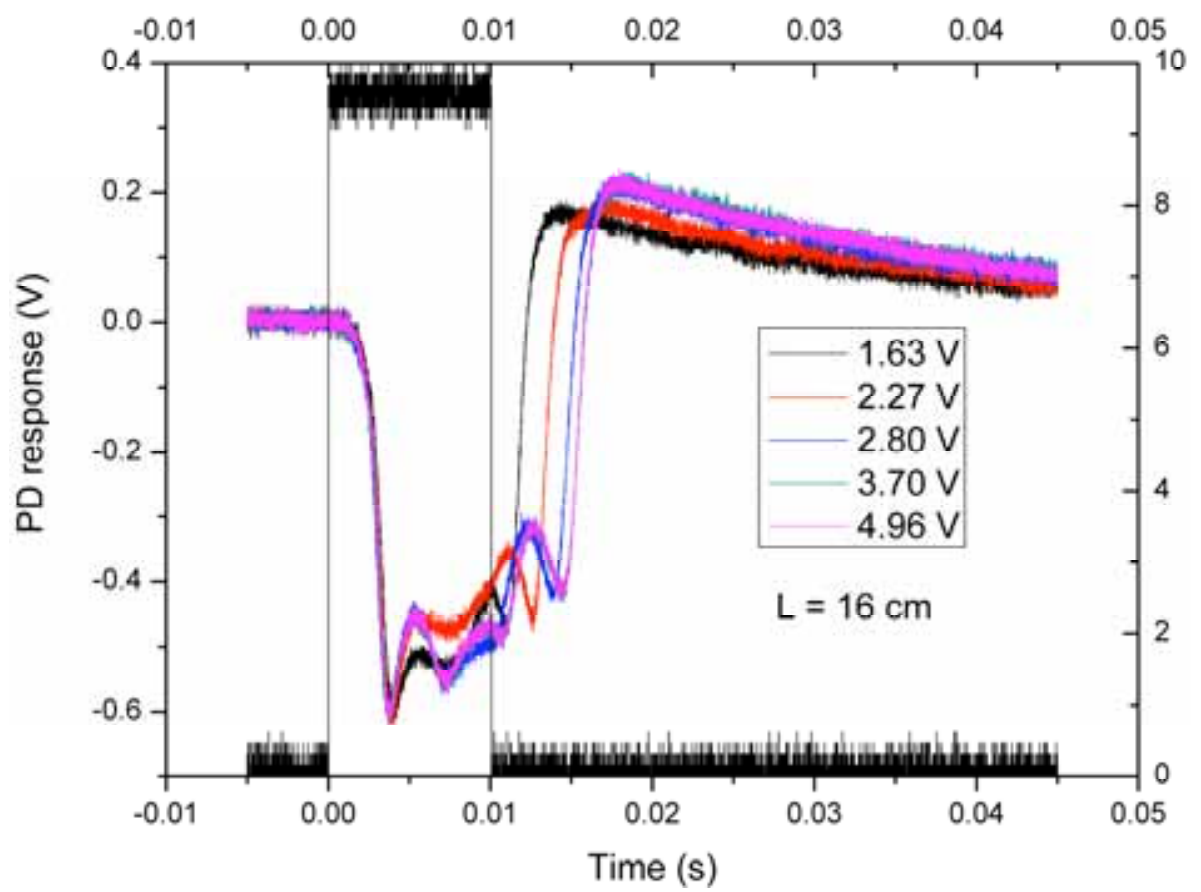

Fig. (3). Time response of a grating submitted to varying input pulse amplitudes at the fixed length $\mathrm{L}=16 \mathrm{~cm}$. For visualization purposes, only one square pulse is shown.

Fig. (3). In this case, the distance L remains fixed, but the average amplitude of the square pulse input to the piezoelectric driver is changed. As the pulse amplitude increases, the strain also increases, leading to the broadening of the optical response.

The delay response and the rise time are the two other features retrieved from Fig. (2). The delay response corresponds to the time interval between the onset of the strain wave in the fiber and the time in which the grating starts to respond. The delay response represents fundamentally the time needed for the strain impulse to reach the grating and cause the Bragg wavelength shift. This definition of delay response is somewhat arbitrary and within the scope of this work is established as the time interval between the onset of the square pulse and the time that corresponds to $10 \%$ of the grating voltage response. In the present configuration, the estimated delay response varies from $1.45 \mathrm{~ms}$ for $\mathrm{L}=16 \mathrm{~cm}$ up to $3.25 \mathrm{~ms}$ for $\mathrm{L}=46 \mathrm{~cm}$.

The rise time corresponds to the time needed for the grating to shift from its rest position to the point where the photo-detector voltage reaches it is maximum value. For the sake of definition, it is computed as the time difference between the $10 \%$ and $90 \%$ of the signal response (voltage) coming from the photodetector and displayed on the oscilloscope. From Fig. (2) one observes different rise times as the distance $\mathrm{L}$ is changed, noting that this time increases as the grating is kept far away from the piezoelectric-driven stage (see the curve for $\mathrm{L}=46 \mathrm{~cm}$ in Fig. (2)). Using this definition one estimates rise times varying from $1.39 \mathrm{~ms}(\mathrm{~L}=$ $16 \mathrm{~cm})$ up to $4.2 \mathrm{~ms}(\mathrm{~L}=46 \mathrm{~cm})$.

The strain applied to the grating can be also estimated once the wavelength shift is measured. Unfortunately, optical spectrum analyzers are not suitable in this case, as their time response is too slow for providing accurate information in a dynamic situation. However, the wavelength shift can be evaluated considering the maximum wavelength shift obtained in the system in a static situation when the piezoelectric device is driven to its maximum acceptable input voltage. The resulting curve is linear and its slope gives the wavelength rate of change per voltage. In the dynamic situation, when the piezo is excited by the square pulse, one is able to read the maximum applied value to the device from a voltage meter. The achieved wavelength shift then follows straightforward. This way, $\Delta \mathrm{L} / \mathrm{L}$ is evaluated using equation (1), where $\gamma=0.787$ for silica fibers.

A relationship can now be established between the optical pulse width observed in the oscilloscope and the applied strain given by $\Delta \mathrm{L} / \mathrm{L}$ (Fig. 4). For this plot, the width was taken considering the Full Width at Half Maximum (FWHM) values of the curves shown in Fig. (3) for $L=16$ $\mathrm{cm}$.

As a final remark, from the curves shown in Fig. (2) and from our definition of delay response one can also estimate the propagation time of the excitation impulse till it reaches the grating. Considering the cases for $\mathrm{L}=16 \mathrm{~cm}$ and $46 \mathrm{~cm}$ and the respective time delay responses, a value of approximately $169 \mathrm{~m} / \mathrm{s}$ is obtained. This result seems to be conditioned by several factors, such as the impulse response of the piezoelectric-driven stage where the fiber is fixed and the glue time delay response, both of which deserve further investigation. However, it is worth mentioning here that in silica fibers directly excited by acoustical waves with frequencies in the order of a few $\mathrm{MHz}$, the speed of the acoustical wave is dictated by the square root of the ratio between the elastic modulus and the material density. For silica fibers 
this velocity is given as $5,740 \mathrm{~m} / \mathrm{s}$ (Tsuda CST 2006) [5] (Beadle IEEE TUFFC 1998) [6].

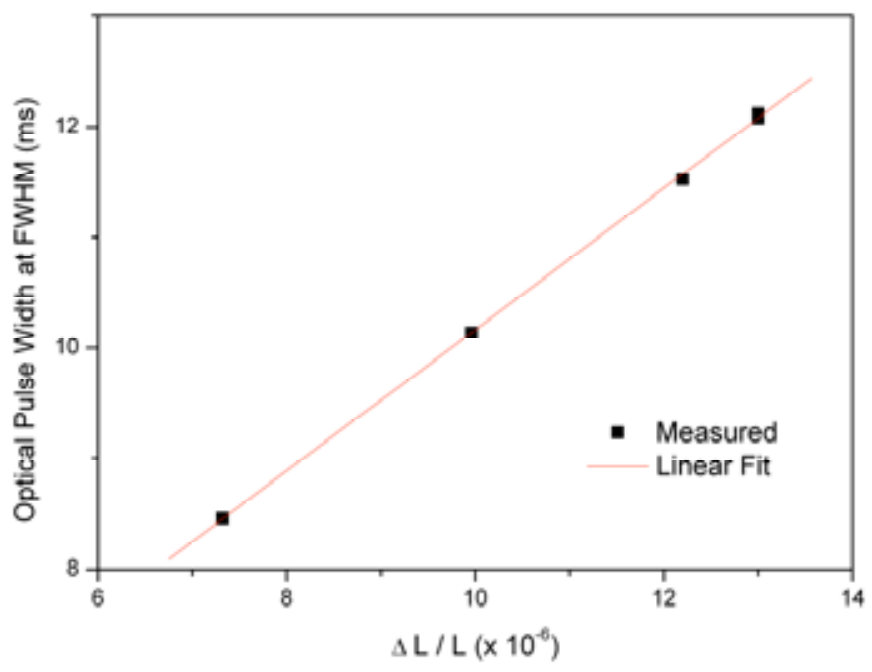

Fig. (4). The plot shows the linear relationship between the pulse width (FWHM) and the strain applied using the piezoelectric-driven stage.

\section{CONCLUSIONS}

From the analysis of the time response of gratings a variety of features can be retrieved, which are of importance for further understanding the operation and fast tuning of devices fabricated in fiber Bragg gratings. Particularly, the de- lay response and the rise time are parameters directly obtained from the excitation of the grating by using a single pulse. The technique is especially important for the design of remote tunable grating based sensors as well as length dependent tunable and fast devices.

\section{ACKNOWLEDGMENTS}

The authors wish to acknowledge the assistance of Michael Stevenson in the fabrication of the gratings. Alexandre Pohl acknowledges the financial support of the Conselho Nacional de Pesquisa e Desenvolvimento (CNPq), Brazil, by means of a research fellowship grant.

\section{REFERENCES}

[1] J. Canning, "New Fibre and Grating Technologies for Laser and Sensors, Frontiers in Laser and Optoelectronics", W. T. Arkin ed., Nova Scientific Publishers, US, 2007.

[2] A. Othonos, and K. Kalli, Fiber Bragg Gratings, Artech House, Norwood, USA, 1999.

[3] A. C. Eringen, and E. S. Suhubi, Elastodynamics - Linear Theory, Academic Press, New York, vol. 2, 1975.

[4] P.T. Neves Jr, and A.A.P. Pohl, "Time Analysis of the Wavelength Shift in Fiber Bragg Gratings", accepted for publication in IEEE/OSA J Ligthwave Technol.

[5] H. Tsuda, "Ultrasound and Damage Detection in CFRP using Fiber Grating Sensors", Composites Sci Technol, vol. 66, p. 676683, 2006.

[6] B.M. Beadle and R. S. Weis, Longitudinal Vibrations of a Silica Fiber Segment Characterized Using a Fiber Bragg Grating, IEEE Transaction on Ultrasonics, Ferroelectrics and Frequency Control, vol. 45, no. 5, p. 1100-1104, September 1998. 